\title{
Clinical Analysis of Patients With New-Onset Untreated Systemic Lupus Erythematosus Complicated With Cytomegalovirus Infection Short Title: Untreated SLE With CMV Infection
}

\section{Xin Li}

Peking University Third Hospital

\section{Shuang Gao}

Peking University Third Hospital

Xiao Li Deng ( 13683657545@163.com )

Peking University Third Hospital

\section{Research Article}

Keywords: new-onset untreated systemic lupus erythematosus, untreated systemic lupus erythematosus, cytomegalovirus infection, cytomegalovirus, antiviral therapy, rheumatology, prognosis

Posted Date: April 12th, 2021

DOl: https://doi.org/10.21203/rs.3.rs-394900/v1

License: (c) (1) This work is licensed under a Creative Commons Attribution 4.0 International License.

Read Full License 
Clinical analysis of patients with new-onset untreated systemic lupus erythematosus complicated with cytomegalovirus infection

Short title: Untreated SLE with CMV infection

Xin $\mathrm{Li}^{1}$, MS; Shuang $\mathrm{Gao}^{2}$, MS; Xiao Li Deng ${ }^{1 *}, \mathrm{MD}$

${ }^{1}$ Medical Science Department Peking University Third Hospital, 49 Huayuan North Road, Haidian District, Beijing 100191, China

${ }^{2}$ Medical Science Department Peking University Third Hospital, 49 Huayuan North Road, Haidian District, Beijing 100191, China

*Corresponding author:

Xiao Li Deng

Peking University Third Hospital, 49 Huayuan North Road, Haidian District, Beijing 100191, China

Tel: 13683657545; Fax: (010)82802529; Email: 13683657545@163.com 


\section{Abstract}

We investigated the clinical characteristics and short-term prognosis of patients with new-onset, untreated systemic lupus erythematosus (SLE) complicated with active cytomegalovirus (CMV) infection. Of the 86 patients enrolled in this study, 60 had active CMV infection. The proportions of patients with fever $(65.0 \%$ versus $38.5 \%, P=0.041)$, hematologic system involvement $(80.0 \%$ versus $57.7 \%, P=0.032)$, kidney involvement $(50.0 \%$ versus $26.9 \%, P=$ $0.047)$, anti-double-stranded-DNA antibody positivity $(51.7 \%$ versus $26.9 \%, P=0.034)$, and severe disease activity (SLEDAI-2000 $\geqslant 15$ points; $58.3 \%$ versus $30.8 \%, P=0.019$ ) were significantly higher, whereas serum $\mathrm{C} 3$ complement levels $(0.48 \pm 0.22$ versus $0.60 \pm 0.25, P$ $=0.032$ ) were significantly lower in the CMV+ group than in the CMV-group, respectively. Initial SLE treatment, clinical features, laboratory data, and SLE treatment did not differ at 3and 6-month follow-ups. In patients with $\mathrm{CMV}+$, there were no significant differences in clinical features, laboratory data, SLE treatment, and laboratory index at 3-month follow-up between patients with or without antiviral treatment. These findings provided important insights into the characteristics of new-onset SLE complicated with active CMV infection and showed that antiviral treatment may have affect short-term prognosis.

Keywords: new-onset untreated systemic lupus erythematosus, untreated systemic lupus erythematosus, cytomegalovirus infection, cytomegalovirus, antiviral therapy, rheumatology, prognosis 


\section{Introduction}

The etiology and pathogenesis of systemic lupus erythematosus (SLE) is unclear. According to recent studies, chronic infections are associated with SLE ${ }^{1}$. Human cytomegalovirus (HCMV, also known as CMV) is a pathogen that mostly infects the immunocompromised population ${ }^{2}$ and is closely related to the occurrence and development of $\mathrm{SLE}^{3,4}$. Antibodies against CMV are commonly detected in patients with SLE. Previous studies reported a high incidence of CMV infection in patients with SLE being treated with steroids and/or immunosuppressants. Most studies have indicated that CMV infection is the result of an immunocompromised status induced by steroid and immunosuppressant therapy. However, some case reports and our findings during clinical practice showed that SLE onset can occur directly after or at the same time as CMV infection. Moreover, the clinical characteristics of patients with new-onset SLE complicated with active CMV infection and the requirement for antiviral treatment in these patients are unclear.

Therefore, we investigated the clinical characteristics of patients with new-onset untreated SLE complicated with active CMV infection and preliminarily observed their short-term prognosis after antiviral treatment.

\section{Results}

\section{Patient profiles}

Eighty-six patients, aged 18-80 years, with new-onset SLE were enrolled in this study; 76 were female $(88.4 \%)$, and 10 were male $(11.6 \%)$. The average age was $34.97 \pm 13.78$ years. The most common clinical manifestations were hematological system involvement (63 cases, $73.3 \%$ ), fever (50 cases, $58.1 \%$ ), and rash (48 cases, $55.8 \%$ ). The numbers of cases with low, moderate, and severe disease activities were $18(20.9 \%), 22(25.6 \%)$, and $43(50.0 \%)$, respectively. Active CMV infection was diagnosed in 60 (69.8\%) cases, among which 29 
(33.7\%) were CMV IgM-positive, one (1.2\%) was CMV DNA-positive, 45 (52.3\%) were CMV pp65-positive, and 27 (31.4\%) were CMV IgM- and CMV IgG-positive. No active CMV infection was found in 26 cases (30.2\%). The demographic and clinical characteristics of these patients are shown in Table 1.

\section{Clinical results}

As shown in Table 2 , the percentages of fever $(65.0 \%$ versus $38.5 \%$, respectively; $P=0.041)$, hematologic system involvement $(80.0 \%$ versus $57.7 \%$, respectively; $P=0.032)$, and kidney damage $(50.0 \%$ versus $26.9 \%$, respectively; $P=0.047)$ were significantly higher in patients in the CMV+ group than in those in the CMV-group. In addition, the SLEDAI-2000 scores of patients with severe disease activity ( $\geqslant 15$ points) were significantly higher in the CMV+ group than in the CMV- group (58.3\% versus $30.8 \%$, respectively; $P=0.019)$. The proportion of anti-double-stranded DNA(dsDNA) antibody-positive ( $>1: 10)$ patients in the $\mathrm{CMV}+$ group was significantly higher than that in the CMV- group (51.7\% versus $26.9 \%$, respectively; $P=$ $0.034)$.

\section{Laboratory results}

As shown in Table 3, serum C3 levels in patients in the CMV+ group $(0.48 \pm 0.22$ versus 0.60 \pm 0.25 , respectively; $P=0.032$ ) were lower than those in the CMV-group. However, other laboratory results showed only minor differences.

Comparison of initial SLE treatment between $C M V+$ and $C M V$-groups

The results of the initial treatment analysis for the two SLE groups are shown in Table 4. The differences in the rates of glucocorticoid pulse therapy $(26.7 \%$ versus $11.5 \%$, respectively; $P=$ 0.120), high-dose glucocorticoid $(45.0 \%$ versus $34.6 \%$, respectively; $P=0.369)$, and glucocorticoids combined with immunosuppressants $(50.0 \%$ versus $34.6 \%$, respectively; $P=$ 0.188) between the CMV+ and CMV-groups were not significant.

Follow-up results 
At the 3-month follow-up, 77 patients were evaluated, among which 23 were in the CMVgroup, and 54 were in the CMV+ group. Ten patients $(18.5 \%, 10 / 54)$ in the CMV+ group did not meet the SLE classification criteria. Differences in the percentages of increased serum complement C3 levels compared with initial serum complement C3 levels (55.4\% versus 47.6\%, respectively; $P=0.544)$ as well as liver transaminitis $(12.5 \%$ versus $28.6 \%$, respectively; $P=0.182$ ) between the $\mathrm{CMV}+$ and $\mathrm{CMV}$ - groups were not significant. For clinical manifestations, the difference in the rate of fever ( $5.4 \%$ versus $19.1 \%$, respectively; $P=0.157$ ) between the $\mathrm{CMV}+$ and $\mathrm{CMV}$ - groups was not significant. In terms of SLE treatment, the $\mathrm{CMV}+$ and CMV- groups showed no significant differences when a small dose of glucocorticoids $(69.6 \%$ versus $90.5 \%$, respectively; $P=0.059)$ and glucocorticoids combined with immunosuppressive agents $(35.7 \%$ versus $28.6 \%$, respectively; $P=0.555)$ were used. Differences in the rates of SLEDAI-2000 scores between the CMV+ and CMV- groups decreased by at least $50 \%(75.0 \%$ versus $76.2 \%$, respectively; $P=0.914)$, and the PGA scores evaluated by physicians $(0.79 \pm 0.92$ versus $0.72 \pm 0.94$, respectively; $P=0.761)$ were not significantly different.

At the 6-month follow-up, 55 patients, including 17 from the CMV-group and 38 from the CMV+ group, were evaluated. Differences in the rates of SLEDAI-2000 scores less than or equal to 4 points $(86.8 \%$ versus $94.1 \%$, respectively; $P=0.740)$ and PGA scores evaluated by physicians $(0.21 \pm 0.64$ versus $0.17 \pm 0.51$, respectively; $P=0.500)$ between the $\mathrm{CMV}+$ and CMV- groups were not significant. For SLE treatment, differences in the rates of unused glucocorticoids (36.1\% versus $26.3 \%$, respectively; $P=0.554)$ and glucocorticoidimmunosuppressant combinations $(38.9 \%$ versus $31.6 \%$, respectively; $P=0.592)$ were not significant.

\section{Results of antiviral and nonantiviral treatments}

Patients with new-onset SLE in the CMV+ group were divided into antiviral and nonantiviral 
treatment groups. The clinical manifestations, laboratory results, initial treatment, medication, and decreased SLEDAI-2000 scores of the two groups were statistically analyzed. As shown in Table 5, the differences in clinical manifestations between the antiviral+ and antiviralgroups, including the incidence of fever $(69.2 \%$ versus $63.8 \%$, respectively; $P=0.974)$, incidence of liver transaminitis $(38.5 \%$ versus $57.5 \%$, respectively; $P=0.225)$, and SLEDAI2000 scores $(16.23 \pm 5.11$ versus $16.23 \pm 7.15$, respectively; $P=0.998)$, were not significant. In the initial SLE treatment, differences in glucocorticoid pulse therapy $(23.1 \%$ versus $27.7 \%$, respectively; $P=0.981)$, high-dose glucocorticoid treatment $(69.2 \%$ versus $38.3 \%$, respectively; $P=0.086)$, and glucocorticoids combined with immunosuppressants $(38.5 \%$ versus $34.0 \%$, respectively; $P=0.974$ ) between the antiviral+ and antiviral- groups were not significant.

At the 3-month follow-up, the differences in the rate of SLEDAI-2000 score less than or equal to 4 points $(33.3 \%$ versus $56.8 \%$, respectively; $P=0.149)$ and the PGA scores evaluated by doctors $(0.92 \pm 0.79$ versus $0.66 \pm 0.86$, respectively; $P=0.355)$ between the antiviral treatment group and non-antiviral treatment group were not significant. In terms of SLE medication, the differences in the rates of low-dose hormone (58.3\% versus $72.7 \%$, respectively; $P=0.544)$ and hormone combined with immunosuppressant $(50.0 \%$ versus $31.8 \%$, respectively; $P=0.196$ ) between the antiviral+ and antiviral- groups were not significant.

\section{Discussion}

In recent decades, many studies have examined the roles of viral infection in the pathogenesis of SLE, also known as the viral hypothesis ${ }^{5}$. CMV belongs to the herpes virus family and is thought to trigger SLE, with CMV DNA and high levels of serum anti-CMV IgM detected in patients with initial symptoms ${ }^{6}$ or disease exacerbation ${ }^{7}$. However, whether CMV infection 
triggers SLE or occurs simultaneously with or after SLE onset remains unclear. Newkirk et al. showed that the U1 small nuclear ribonucleoprotein (U1 snRNP) antibody is related to CMV antibody in the serum of patients with $\mathrm{SLE}^{8}$. An earlier study by Mohamed et al. suggested that the positive rate of anti-HCMV antibodies is higher in patients with SLE than in healthy individuals ${ }^{9}$. Stratta et al. found that HCMV infection is inseparable from the "vascular" manifestations of SLE and is related to SLE with nephrotic syndrome ${ }^{10}$. Nawata et al. also confirmed that HCMV infection can lead to the occurrence of SLE ${ }^{11}$. These conclusions suggest that the onset of SLE is induced by active CMV infection.

Our study showed that the incidence of active CMV infection in patients with new-onset SLE was $69.8 \%$, which was relatively high. A review of previous studies revealed differences in the reported proportion of patients with SLE and CMV infection. The reasons for these discrepancies may be related to the definition of active CMV infection, material obtained, and selection of samples. Takizawa et al. ${ }^{12}$ used the CMV pp65 antigen to detect active CMV infection; they found that the highest incidence of CMV infection was observed in patients with SLE $(n=51)$ and that $75 \%$ of CMV-infected patients presented with CMV disease. Rozenblyum ${ }^{13}$ improved CMV etiology examination using urine and bronchoalveolar lavage fluid and found that the active CMV infection rate in patients with SLE was 1.04\%. Through neutralization of the RF factors that may interfere with CMV detection, $\mathrm{Su}{ }^{14}$ found that nine of 87 patients with SLE were positive for CMV IgM, giving a positive rate of $10.34 \%$. However, these methods have the disadvantages of a long separation cycle and difficulty in obtaining materials, making early detection difficult. An isolated negative result cannot exclude the possibility of active CMV infection, and as a result, these tests cannot be easily conducted in clinical practice.

The definition of active CMV infection thus includes CMV DNA positivity $\left(>5 \times 10^{2}\right.$ copy number/mL); CMV pp65 positivity ( $>5 \times 10^{5}$ white blood cells $\left./ \mathrm{mL}\right)$; CMV IgM- 
positivity $(>30 \mathrm{U} / \mathrm{mL})+\mathrm{CMV} \operatorname{IgG}$ positivity $(>1 \mathrm{U} / \mathrm{mL})$; a titer of serum antibody CMV IgG, obtained by double testing, greater than or equal to 4-fold higher than the baseline data or positive for both CMV IgM and CMV IgG, indicating recent active infection. Notably, one isolated contradictory result cannot exclude infection. This definition can improve the sensitivity of active CMV infection screening, but may relatively reduce specificity. This may be one of the reasons for the higher proportion of active CMV infections identified in this study than those in the previous studies, with patient selection being another. Most previous studies analyzed all cases of patients with SLE and CMV infection, and only a few cases were reported for patients with new-onset SLE and CMV infection. A larger sample size and more experiments are needed to determine the infection rate of new-onset SLE with active CMV infection.

The reasons for the significance of differences in clinical manifestations and laboratory test results in groups with new-onset SLE with or without active CMV infection are described below. Earlier studies reported that in addition to conventional fever, cytopenia, and liver function damage, patients with SLE and active CMV infection often present with involvement of organs and the presence of interstitial pneumonia, hemorrhagic alveolitis, and retinitis, resulting in death in severe cases. As an environmental factor promoting SLE ${ }^{15}{ }^{16}$, CMV may initiate immune responses through various mechanisms, such as molecular simulation, epitope expansion, and stimulation of polyclonal B cell activation, participating in the pathogenesis of SLE ${ }^{17}$. Long-term use of glucocorticoids and immunosuppressants in patients with SLE leads to further activation of CMV, which aggravates SLE disease activity and results in a vicious circle $^{18}$.

Autoantibodies are commonly observed in patients infected with CMV. For example, antiphospholipid and anti-CD13 antibodies may be observed in patients with CMV-bone marrow transplantation ${ }^{19}$. Anti-CD13 antibodies are related to the development of chronic graft-versus- 
host disease. Among the receivers of solid organ transplantation, non-organ-specific autoantibodies, such as anti-endothelial cells, anti-sm, and anti-nuclear antibodies are related to $\mathrm{CMV}$ infection ${ }^{20}$. CMV may increase the risk of rejection mediated by human immunity and chronic graft-versus-host disease, following allogeneic grafts ${ }^{21}$. In addition, increased generation of hypergammaglobulinemia, cryoglobulinemia, and autoantibodies is observed on monocytes induced by CMV and post-reperfusion syndrome ${ }^{22,23}$. Anti-dsDNA antibodies are SLE-specific antibodies that act as markers of SLE disease activities ${ }^{24}$. Previous research has suggested that after obtaining pp65 immunity, C57BL/6 mice show a significant rise in antidsDNA antibody levels; therefore, CMV may be related to the generation of anti-dsDNA ${ }^{25}$. CMV infection may induce SLE-related morbidity or cause variations in the illness state. According to previous research ${ }^{26}$, U1 snRNP, an important target antigen for autoimmune diseases, is involved in the process of mRNA maturity as a type of small nuclear ribonucleoprotein. In the hematologic system of patients with SLE, anti-CMV antibodies are associated with anti-U1 snRNP antibodies. However, in the serum of patients with other connective tissue diseases, anti-CMV does not associate with U1 snRNP. Thus, CMV is an important element that induces patients with SLE to generate an autoimmune reaction. Consistent with these results, we found that the incidence of anti-dsDNA antibody-positivity in the CMV+ group was higher than that in the CMV-group.

There may be several reasons for the higher proportion of hematologic system involvement in the CMV+ group than that in the CMV- group in this study. First, like most herpes viruses, CMV has latent-activated biological characteristics. Once CMV invades the human body, most people will have latent infection without symptoms. When active CMV infection occurs, the virus first replicates in peripheral blood mononuclear cells and then invades the phagocytic cells of other organs ${ }^{27}$; therefore, the hematologic system is first involved when CMV replication is active. Second, a crossreaction occurs between the anti- 
platelet and anti-CMV antibodies, resulting in thrombocytopenia. Shigemura proposed that different fever levels cannot discriminate between CMV infection and SLE; however, thrombocytopenia is mainly present in patients infected with $\mathrm{CMV}^{28}$. In addition, we showed that patients with new-onset SLE and active CMV infection were more likely to have symptoms of kidney damage, possibly because CMV may be dormant in the kidney for a long time and only invade the kidney tissue once activated ${ }^{29}$.

Patients with new-onset SLE and active CMV infection may have several specific symptoms, such as fever, hematologic system damage, and kidney damage. However, overall, the clinical manifestations of an active CMV infection are very similar to those of SLE. At the 3-month follow-up visit in the CMV+ and CMV-groups, 10 patients $(18.5 \%, 10 / 54)$ in the CMV+ group did not meet the SLE classification criteria; this may be explained by several reasons. First, in the Hopkins study ${ }^{30}$, according to the DORIS definition, the median time for complete remission and clinical remission of SLE was 3 months, and the median duration of lupus low disease activity state was 1.6 years. Accordingly, the above 10 patients with SLE may have achieved clinical remission or complete remission. Another possibility is that these 10 patients had active CMV infection in the early stage, but because the clinical symptoms of SLE and CMV were similar, they were included in the study after they had met the SLE classification criteria at the time of diagnosis of SLE. Previous studies showed ${ }^{31}$ that if CMV is active, the clinical symptoms can be relieved after 21 days of intravenous antiviral treatment. However, in our subsequent experiments in which the CMV+ group was subdivided into antiviral treatment and non-antiviral treatment groups, no significant differences were observed between the two groups in terms of clinical symptoms and laboratory test results. This result indicated that antiviral therapy had no significant effect on the short-term prognosis of patients with SLE. Moreover, previous studies ${ }^{32,33}$ found no correlation between CMV IgM and SLEDAI scores. Although the above-mentioned 10 patients with SLE may have achieved clinical remission, the 
number of patients enrolled in this study was small, making it difficult to adequately distinguish between them; a larger sample size is needed for verification, along with laboratory parameters or further pathological examinations, to confirm this assumption.

As stated, patients in the CMV+ group were further divided into two groups: those receiving antiviral therapy and those not receiving antiviral therapy. At follow-up, the results obtained for the antiviral+ and antiviral- groups in this study were consistent with those of previous studies ${ }^{34}$. Moreover, antiviral therapy has been shown to be nonessential for treating active CMV infection complicated with incipient SLE. In addition, with the remission of SLE, the CMV virus marker can give a negative result without intervention ${ }^{35}$. It is thought that the death rate of patients with SLE and active CMV infection is not high, regardless of high morbidity ${ }^{36}$; therefore, antiviral therapy is not necessary. With remission of SLE, the CMV infection marker can spontaneously change from positive to negative ${ }^{37}$. On study ${ }^{38}$ showed that inflammation itself was important for the persistence of CMV infection and that controlling inflammation could facilitate active CMV infection. During follow-up visits with the group to which antiviral therapy was administered and the group that did not receive antiviral therapy, no significant differences in clinical symptoms and laboratory results were identified. This indicates that controlling inflammation with steroid and immunosuppressant drugs is more important than antiviral treatment for controlling both SLE and CMV infection.

This was a single-center study. Considering the limited time and small number of samples, we could not fully summarize the clinical characteristics of the combination of new-onset SLE with active CMV infection; therefore, further investigations are needed to verify our results.

\section{Conclusions}

Patients with new-onset SLE and active CMV infection were more likely to exhibit fever, hematologic system involvement, kidney damage, decreased serum complement $\mathrm{C} 3$, and 
positive anti-dsDNA antibodies than those without active CMV infection. In addition, in the CMV+ group, the SLEDAI-2000 score showed a higher proportion of severe disease activity (58.3\%). Active CMV infection may be involved in the pathogenesis or exacerbation of SLE; however, antiviral therapy may have no significant effect on the treatment and short-term prognosis of SLE. This study provides data supporting the role of active CMV infection in the pathogenesis of SLE.

\section{Methods}

\section{Characteristics of the patients}

This was a retrospective study in which 86 patients with new-onset untreated SLE, confirmed at the Peking University Third Hospital from 2011 to 2018, were enrolled. The study protocol was approved by the Ethics Committee of the Peking University Third Hospital (IRB00006761-M2019077) and conformed to the most recent provisions of the Declaration of Helsinki. All patients signed informed consent forms prior to being included in the study. The inclusion criteria were as follows: 1) patients who met the classification criteria for SLE revised by the American College of Rheumatology in 1997, within 1 week after diagnosis and 2) patients who had never used glucocorticoids or immunosuppressants. Exclusion criteria were as follows: 1) patients aged $<18$ or $>80$ years; 2) patients who had other circulatory system disorders and malignant tumors; and 3) patients with other bacterial, fungal, or Epstein-Barr virus infections.

\section{CMV laboratory testing}

CMV DNA detection

Quantitative detection of CMV DNA was performed using real-time quantitative polymerase chain reaction with fluorescence probing (CMV nucleic acid quantitative detection kit; Da An Gene Co., Ltd., Sun Yat-sen University). The positive criterion was $5 \times 10^{2}$ copies $/ \mathrm{mL}$. 


\section{CMV pp65 detection}

Monoclonal antibody indirect immunofluorescence (CMV Brite kit, IQ; DL Groningen, The Netherlands) was used to detect the pp65 antigen in human peripheral blood polymorphonuclear leukocytes. Only the CMV pp65 antigen results were reported qualitatively. A positive result was defined as $2 \times 10^{5}$ polymorphonuclear leukocytes.

CMV-specific IgM and IgG detection

Peripheral blood CMV-specific IgM and IgG antibodies were detected via enzyme-linked immunosorbent assays (ETI-CYTOK-M reverse PLUS kit, P002035; DiaSorin, Saluggia, Italy).

Definition of active CMV infection

Active CMV infection was defined as the presence of any of the following: CMV DNA in peripheral blood $\left(>5 \times 10^{2}\right.$, copy number $/ \mathrm{mL}$ ); CMV pp65 antigen in the blood (cell number/2

$\times 10^{5}$ leukocytes); CMV IgM antibody $(>30 \mathrm{U} / \mathrm{mL})+\mathrm{CMV} \operatorname{IgG}$ antibody $(>1 \mathrm{U} / \mathrm{mL})$. The titer of serum antibody CMV IgG, obtained by double testing, was at least 4-fold higher than that of the baseline data or both CMV IgM and CMV IgG were positive, indicating recent active infection. Patients were divided into two groups according to their active CMV infection status: those with active CMV infection were placed in the CMV+ group, and those with no active CMV infection were placed in the CMV- group.

\section{Laboratory tests}

Laboratory tests mainly included routine blood, biochemical, and urine tests. Other parameters included related antibodies, such as anti-dsDNA antibodies, anti-smooth muscle cell (anti-sm) antibodies, and immunoglobulins.

\section{Treatment for active CMV infection}

Patients with new-onset untreated SLE and CMV infection were further divided into antiviral and non-antiviral therapy groups according to their symptoms, laboratory examination results, 
clinician judgment, and patient wishes. The antiviral therapy group, in addition to treatment for SLE, was also intravenously administered ganciclovir as an antiviral treatment for 14 days (5 $\mathrm{mg} / \mathrm{kg}, \mathrm{q} 12 \mathrm{~h}$ ), after which patients continued to take ganciclovir for 14 days ( $1 \mathrm{~g}$ tid). Thus, the course of treatment lasted for 28 days.

\section{Follow-up}

Patients were followed up as outpatients for 3 and 6 months after treatment. Changes in the SLEDAI-2000 score, medication, clinical symptoms, and laboratory results were analyzed. According to the clinical symptoms and laboratory results, the PGA score $(0,1,2-2.5,3)$ was assessed by the same rheumatologist. The results were evaluated and recorded during the follow-up visits and classified as remission, low disease activity, moderate disease activity, and severe disease activity.

Statistical analysis

Statistical analyses were performed using the SPSS software version 21.0 (SPSS, Inc., Chicago, IL, USA). Count results were analyzed by the chi-square or Fisher's exact tests; measurement data were expressed as means \pm standard deviations, and $t$-tests were used for analyses. Results with $P$ values less than 0.05 were statistically significant.

\section{References}

1. Zandman-Goddard, G., et al. Environment and lupus-related diseases. Lupus. 21, 241-250 (2012).

2. Soderberg-Naucler, C. Autoimmunity induced by human cytomegalovirus in patients with systemic lupus erythematosus. Arthritis Res. Ther. 14, 101 (2012).

3. Guo, G., et al. The cytomegalovirus protein US31 induces inflammation through monomacrophages in systemic lupus erythematosus by promoting NF-kappaB2 activation. Cell 
Death Dis. 9, 104 (2018).

4. Eisenstein, E. M. \& Wolf, D. G. Cytomegalovirus infection in pediatric rheumatic diseases: a review. Pediatr. Rheumatol. Online J. 8, 17 (2010).

5. Ramos-Casals, M. Viruses and lupus: the viral hypothesis. Lupus. 17, 163-165 (2008).

6. Rigante, D. \& Esposito, S. Infections and systemic lupus erythematosus: binding or sparring partners? Int. J. Mol. Sci. 16, 17331-17343 (2015).

7. Doaty, S., et al. Infection and lupus: which causes which? Curr. Rheumatol. Rep. 18, 13 (2016).

8. Newkirk, M. M., van Venrooij, W. J., \& Marshall, G. S. Autoimmune response to U1 small nuclear ribonucleoprotein (U1 snRNP) associated with cytomegalovirus infection. Arthritis Res. 3, 253-258 (2001).

9. Mohamed, A. E., et al. Real-Time PCR of cytomegalovirus and Epstein-Barr virus in adult Egyptian patients with systemic lupus erythematosus. Int. J. Rheum. Dis. 18, 452-458 (2015). 10. Stratta, P., et al. Correlation between cytomegalovirus infection and Raynaud's phenomenon in lupus nephritis. Nephron. 82, 145-154 (1999).

11. Nawata, M., et al. Possible triggering effect of cytomegalovirus infection on systemic lupus erythematosus. Scand. J. Rheumatol. 30, 360-362 (2001).

12. Takizawa, Y., et al. Clinical characteristics of cytomegalovirus infection in rheumatic diseases: multicentre survey in a large patient population. Rheumatology.47, 1373-1378 (2008). 13. Rozenblyum, E. V., et al. Cytomegalovirus in pediatric systemic lupus erythematosus: prevalence and clinical manifestations. Lupus. 24, 730-735 (2015).

14. Su, B. Y. J., et al. Incidental discovery of high systemic lupus erythematosus disease 
activity associated with cytomegalovirus viral activity. Med. Microbiol. Immunol. 196, 165170 (2007).

15. Doria, A., et al. Infections as triggers and complications of systemic lupus erythematosus. Autoimmun. Rev. 8, 24-28 (2008).

16. Ramos-Casals, M., et al. Clinical guidelines for the management of acute viral infections in patients with systemic lupus erythematosus. Minerva Med. 100, 437-446 (2009).

17. Soderberg-Naucler, C. Autoimmunity induced by human cytomegalovirus in patients with systemic lupus erythematosus. Arthritis Res. Ther. 14, 101 (2012).

18. Esposito, S., et al. Infections and systemic lupus erythematosus. Eur. J. Clin. Microbiol. Infect. Dis. 33, 1467-1475 (2014).

19. Mengarelli, A., et al. High levels of antiphospholipid antibodies are associated with cytomegalovirus infection in unrelated bone marrow and cord blood allogeneic stem cell transplantation. Br. J. Haematol. 108, 126-131 (2000).

20. Varani, S., et al. Autoantibody appearance in cytomegalovirus-infected liver transplant recipients: correlation with antigenemia. J. Med. Virol. 66, 56-62 (2002).

21. Dubel, L., et al. High incidence of antitissue antibodies in patients experiencing chronic liver allograft rejection. Transplantation. 65, 1072-1075 (1998).

22. Cunha, B. A. \& Chawla, K. Fever of unknown origin (FUO): CMV infectious mononucleosis or lymphoma? Eur. J. Clin. Microbiol. Infect. Dis. 37, 1373-1376 (2018).

23. Mathew, P., et al. T-gamma gene rearrangement and CMV mononucleosis. Am. J. Hematol. 66, 64-66 (2001).

24. Ahlin, E., et al. Autoantibodies associated with RNA are more enriched than anti-dsDNA 
antibodies in circulating immune complexes in SLE. Lupus. 21, 586-595 (2012).

25. Chang, M., et al. Human cytomegalovirus pp65 lower matrix protein: a humoral immunogen for systemic lupus erythematosus patients and autoantibody accelerator for NZB/W F1 mice. Clin. Exp. Immunol. 143, 167-179 (2006).

26. Yoon, G. S., et al. Cytomegalovirus prostatitis: a series of 4 cases. Int. J. Surg. Pathol. 18, 55-59 (2010).

27. Stocchi, R., et al. Management of human cytomegalovirus infection and disease after allogeneic bone marrow transplantation. Haematologica. 84, 71-79 (1999).

28. Shigemura, T., et al. Prevention of transfusion-transmitted cytomegalovirus infection using leukoreduced blood components in patients receiving seronegative umbilical cord blood transplantation. Transfusion. 59, 3065-3070 (2019).

29. Zhou, W. J. \& Yang, C. D. The causes and clinical significance of fever in systemic lupus erythematosus: a retrospective study of 487 hospitalised patients. Lupus. 18, 807-812 (2019). 30. Wilhelm, T. R., Magder, L. S., \& Petri, M. Remission in systemic lupus erythematosus: durable remission is rare. Ann. Rheum. Dis. 76, 547-553 (2017).

31. Asberg, A., et al. Oral valganciclovir is noninferior to intravenous ganciclovir for the treatment of cytomegalovirus disease in solid organ transplant recipients. Am. J. Transplant. 7, 2106-2113 (2007).

32. Bendiksen, S., et al. A longitudinal study of human cytomegalovirus serology and viruria fails to detect active viral infection in 20 systemic lupus erythematosus patients. Lupus. 9, 120126 (2000).

33. Dubey, S., et al. Cytomegalovirus in Indian systemic lupus erythematosus patients: 
troublemaker or onlooker? Pan. Afr. Med. J. 37, 38 (2020).

34. Zhang, L., et al. Cytomegalovirus infection in patients with lupus nephritis: clinical and laboratory features and therapeutic considerations. Clin. Exp. Med. 17, 467-475 (2017).

35. Sekigawa, I., et al. Cytomegalovirus infection in patients with systemic lupus erythematosus. Clin. Exp. Rheumatol. 20, 559-564 (2002).

36. Fujimoto, D., et al. Risk factors associated with elevated blood cytomegalovirus pp65 antigen levels in patients with autoimmune diseases. Mod. Rheumatol. 23, 345-350 (2013).

37. Hrycek, A., et al. Human cytomegalovirus in patients with systemic lupus erythematosus. Autoimmunity. 38, 487-491 (2005).

38. Mansfield, S., et al. Sepsis and cytomegalovirus: foes or conspirators? Med. Microbiol. Immunol. 204, 431-437 (2015).

\section{Author contributions}

DXL conceived and designed the study. LX searched the literature, screened, and selected eligible studies; extracted data and conducted quality assessment; analyzed and interpreted the data; and wrote the manuscript draft. All other authors critically reviewed the manuscript. All authors agree to be accountable for all aspects of the study and ensuring that questions related to the accuracy or integrity of any part of the study are appropriately investigated and resolved. All authors take full responsibility for the integrity of the study and approved the final manuscript as submitted.

\section{Acknowledgments}

We are grateful to all the patients with SLE who participated in this study. 


\section{Funding}

This work was supported by the Project of the National Natural Science Foundation of China (grant no. 81501390). 
Table 1. Patient demographic data

Features

Values, n (\%)

Female

$76(88.4)$

Clinical symptoms

Fever $^{\mathrm{a}}$

$50(58.1)$

Rash

$48(55.8)$

Photosensitivity

$16(18.6)$

Oral ulcer

$17(19.8)$

Hematological system involvement ${ }^{\mathrm{b}}$

$63(73.3)$

Respiratory system involvement ${ }^{\mathrm{c}}$

$39(45.4)$

Kidney involvement ${ }^{\mathrm{d}}$

$37(43.0)$

Digestive system involvement $\mathrm{e}^{\mathrm{e}}$

$41(47.4)$

Neurological complication ${ }^{\mathrm{f}}$

$12(13.9)$

SLEDAI-2000 score (0-4 points)

$3(3.5)$

SLEDAI-2000 score (5-9 points)

$18(20.9)$

SLEDAI-2000 score (10-14 points)

$22(25.6)$

SLEDAI-2000 score ( $\geqslant 15$ points) no.

$43(50.0)$

$\mathrm{RF}^{\mathrm{g}}$ factor-positive

$21(24.4)$

$\underline{\text { CMV infection laboratory data }}$

CMV active infection

$60(69.8)$

CMV IgM-positive

$29(33.7)$

CMV IgG-positive

$62(72.1)$ 


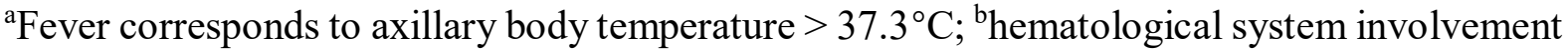

refers to white blood cells $<3.5 \times 10^{9} / \mathrm{L}$, hemoglobin $<115 \mathrm{~g} / \mathrm{L}$, and platelets $<125 \times 10^{9} / \mathrm{L}$.

Any one of the three items that meet the above criteria represents hematological system involvement; 'respiratory system involvement includes pleural effusion, pleurisy, and interstitial lung disease; ${ }^{\mathrm{d}}$ kidney involvement refers to urinary protein $>0.5 / 24 \mathrm{~h}$ or +++ , which needs to be excluded due to insufficient intake or drugs; ${ }^{e}$ digestive system involvement includes liver function (elevated liver enzymes such as ALT and AST) and mesenteric vasculitis; fneurological complications need to exclude drugs and metabolic disorders, recent occurrence of convulsions, disorientation, and cerebrovascular accidents; ${ }^{\mathrm{g}}$ rheumatoid factor 
Table 2. Comparison of clinical and laboratory results between the CMV+ and CMV-groups

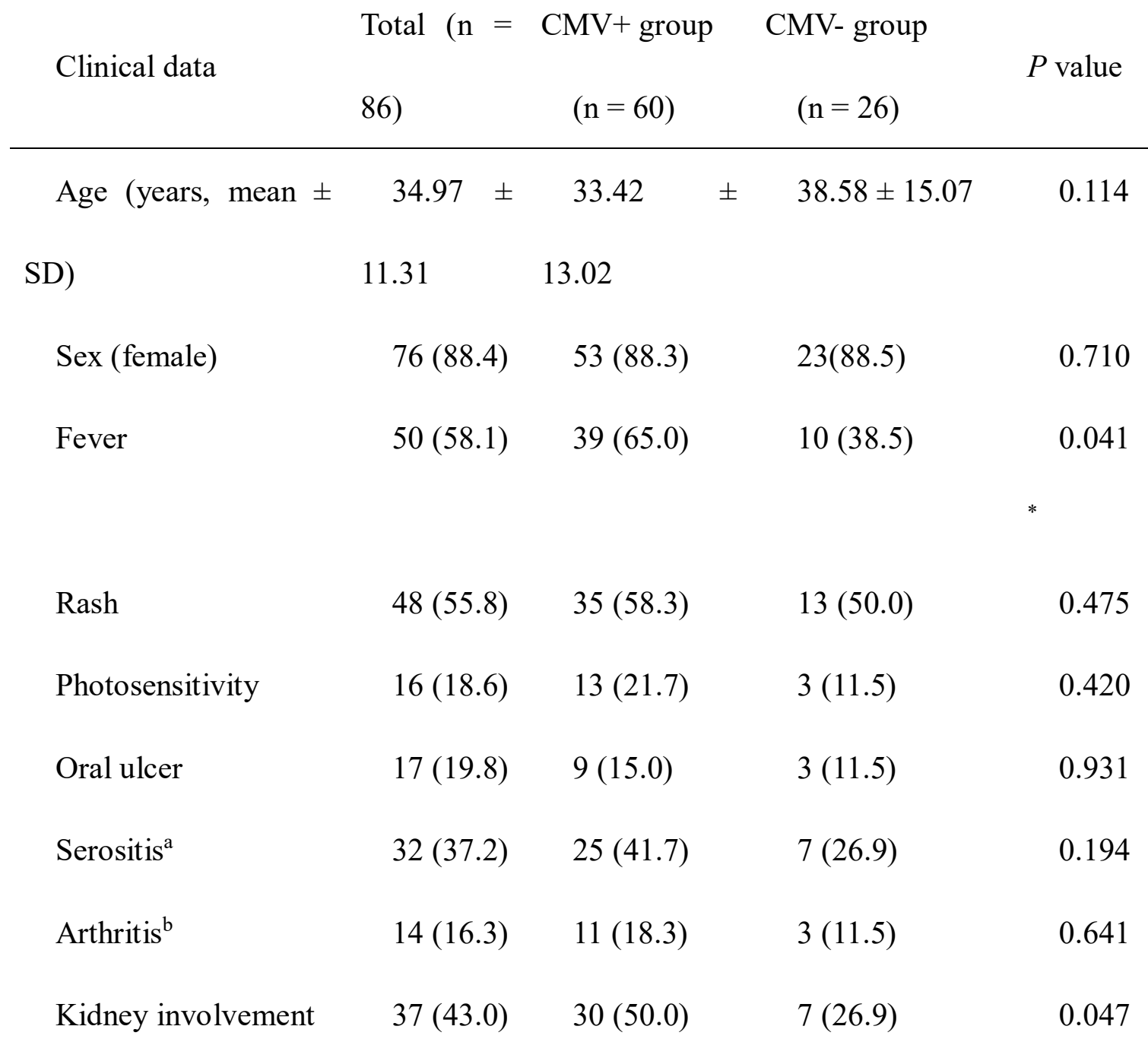

Hematologic system $63(73.3) \quad 48(80.0) \quad 15(57.7) \quad 0.032$

involvement

Digestive system

involvement

$41(47.7) \quad 32(53.3) \quad 9(34.6) \quad 0.11$

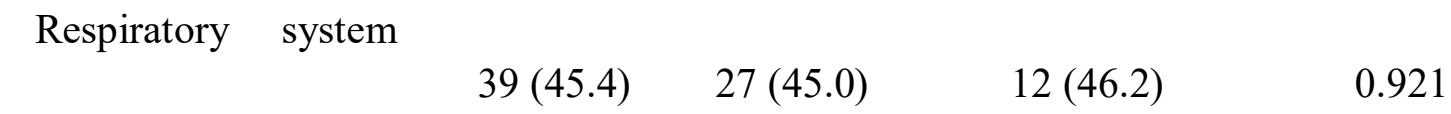

involvement

Neurological

$12(13.9) \quad 10(16.7) \quad 2(7.7)$

0.445 
complications

$\begin{array}{llllll}\text { SLEDAI-2000 } & \text { score } & & & & \\ & & 3(3.5) & 2(3.3) & 1(3.9) & 1 \\ (0-4 \text { points }) & & & & & \\ \text { SLEDAI-2000 } & \text { score } & & & & \\ & & 18(20.9) & 11(18.3) & 7(26.9) & 0.368\end{array}$

(5-9 points)

SLEDAI-2000 score $22(25.6) \quad 13(21.7) \quad 9(34.6) \quad 0.206$

(10-14 points)

SLEDAI-2000 score

$(\geqslant 15$ point $)$

$43(50.0) \quad 35(58.3)$

$8(30.8)$

0.019

Laboratory results

ANA antibody titer > $64(74.4) \quad 45(75.0) \quad 19(73.1) \quad 0.851$

$1: 160$

Anti-ds-DNA ${ }^{c}>1: 10$ $38(44.2) \quad 31(51.7) \quad 7(26.9) \quad 0.034$

cases

$\begin{array}{lcccc}\text { Anti-sm positive } & 23(26.7) & 18(30.0) & 5(19.2) & 0.300 \\ \text { Anti-SSA positive } & 46(53.5) & 34(56.7) & 12(46.2) & 0.369 \\ \text { Anti-SSB positive } & 16(18.6) & 13(21.7) & 3(11.5) & 0.419 \\ \text { Anti-RNP } / \text { sm } & 40(46.5) & 30(50.0) & 10(38.5) & 0.324\end{array}$

positive

$\begin{array}{lllll}\text { Anti-Jo-1 positive } & 0 & 0 & 0 & 1 \\ \text { Anti-Scl-70 positive } & 1(1.2) & 0 & 1(3.9) & 0.302\end{array}$




\begin{tabular}{ccccc}
\hline Anti-rRNP positive & $23(26.7)$ & $17(28.3)$ & $6(23.1)$ & 0.613 \\
RF factor-positive & $21(24.4)$ & $17(28.3)$ & $4(15.4)$ & 0.199 \\
\hline
\end{tabular}

${ }^{*} P<0.05$ was considered significant; ${ }^{\text {a }}$ serositis, presence of pericardial effusion, pleural effusion, or peritoneal effusion; ${ }^{b}$ arthritis: joints are often exhibit tenderness, swelling, or fluid accumulation, and non-erosive arthritis can easily involve two or more peripheral arthritis. Numbers within parentheses are percentages. ${ }^{c}$ Anti-ds-DNA $>1: 10$ : The antibody titer of antids-DNA is equal to or greater than 1:10, showing a positive result. 
Table 3. Comparison of laboratory data between the CMV+ and CMV-groups

\begin{tabular}{|c|c|c|c|}
\hline Laboratory data & $\begin{array}{l}\mathrm{CMV}+\text { group } \\
(\text { mean } \pm \mathrm{SD})\end{array}$ & $\begin{array}{l}\text { CMV- group } \\
(\text { mean } \pm \mathrm{SD})\end{array}$ & $P$ value \\
\hline White blood cells $\left(10^{9} / \mathrm{L}\right)$ & $4.51 \pm 2.49$ & $5.39 \pm 2.64$ & 0.882 \\
\hline Hemoglobin (g/L) & $104.10 \pm 20.91$ & $104.84 \pm 23.31$ & 0.887 \\
\hline Platelet $\left(10^{9 /} \mathrm{L}\right)$ & $154.86 \pm 93.64$ & $199.80 \pm 107.08$ & 0.058 \\
\hline $\mathrm{ALT} u / \mathrm{L}^{\mathrm{a}}$ & $50.27 \pm 75.82$ & $30.65 \pm 24.24$ & 0.229 \\
\hline $\mathrm{AST} u / \mathrm{L}^{\mathrm{b}}$ & $54.25 \pm 74.56$ & $29.57 \pm 13.78$ & 0.120 \\
\hline $\mathrm{C} 3 \mathrm{~g} / \mathrm{L}^{\mathrm{c}}$ & $0.48 \pm 0.22$ & $0.60 \pm 0.25$ & $0.032^{*}$ \\
\hline $\mathrm{C} 4 \mathrm{~g} / \mathrm{L}^{\mathrm{d}}$ & $0.08 \pm 0.57$ & $0.11 \pm 0.06$ & 0.071 \\
\hline
\end{tabular}

${ }^{*} P<0.05$ was considered significant; ${ }^{\mathrm{a} A L T}$, alanine aminotransferase, normal range according to our hospital: $7-40 \mu / \mathrm{L}$; ${ }^{\mathrm{b}} \mathrm{AST}$, aspartate aminotransferase, normal reference range according to our hospital: $13-35 \mu / \mathrm{L}$; ${ }^{\mathrm{c}} \mathrm{C} 3$, complement $\mathrm{C} 3$, normal reference range: $0.85-2 \mathrm{~g} / \mathrm{L}$; ${ }^{\mathrm{d}} \mathrm{C} 4$, complement $\mathrm{C} 4$, normal reference range: $0.12-0.4$. g/L 
Table 4. Comparison of initial SLE treatment between the CMV+ and CMV-groups $(\mathrm{n}=86)$

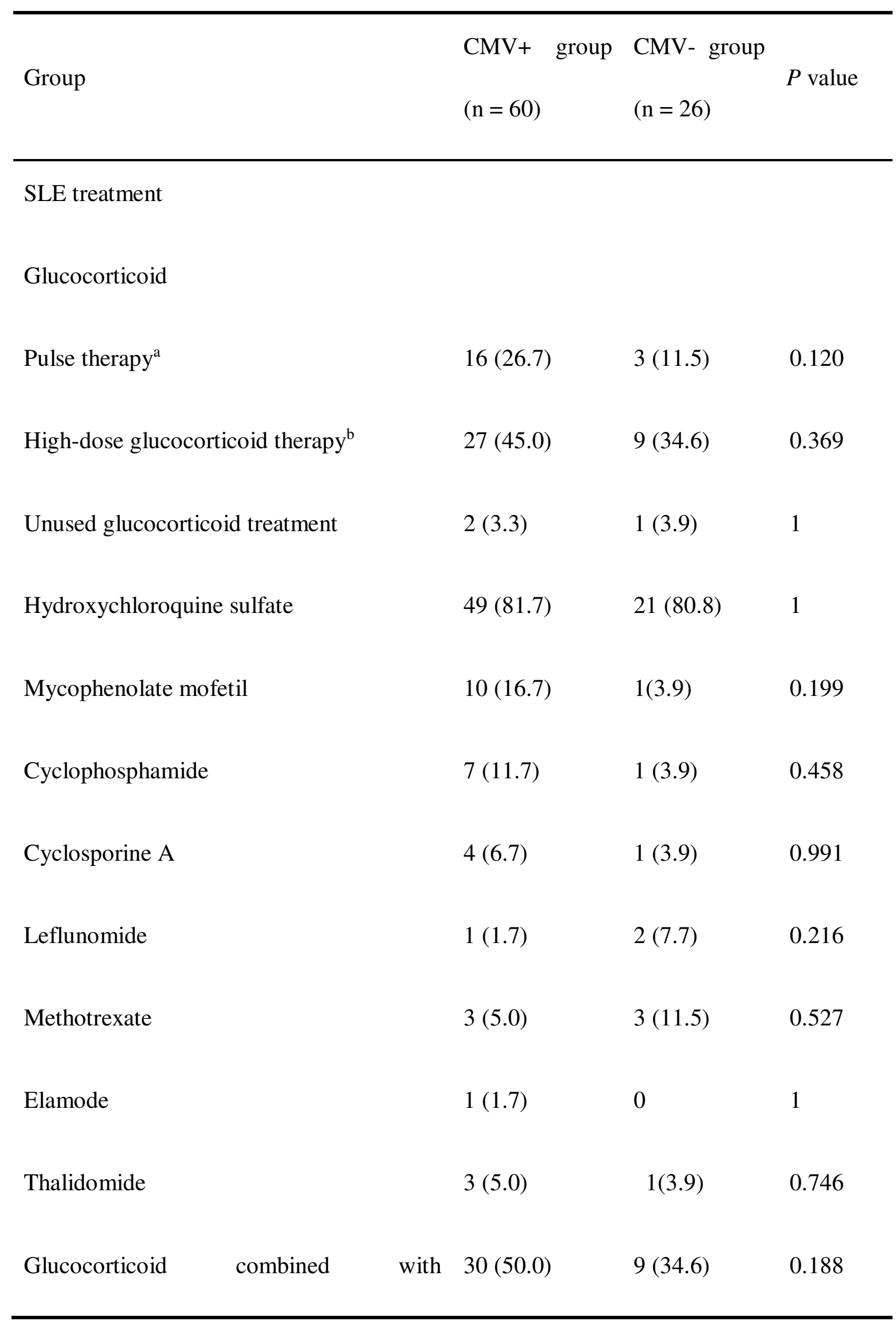


immunosuppressive agent

${ }^{*} P<0.05$ was considered significant; ${ }^{a}$ glucocorticoid pulse therapy is defined as the use of methylprednisolone 200, 500, and $1000 \mathrm{mg} / \mathrm{dL}$; ${ }^{b}$ high-dose glucocorticoid is defined as a glucocorticoid daily dosage of $1-1.5 \mathrm{mg} / \mathrm{kg}$. 
Table 5. Comparison of baseline results between the antiviral and non-antiviral treatment groups

\begin{tabular}{|c|c|c|c|}
\hline & Antiviral & Non-antiviral & \\
\hline Group & treatment & treatment & $P$ value \\
\hline & $\operatorname{group}(n=13)$ & group $(n=47)$ & \\
\hline Age (years, mean $\pm \mathrm{SD}$ ) & $34.00 \pm 15.33$ & $32.84 \pm 12.42$ & 0.781 \\
\hline Sex (female) & $11(84.6)$ & $41(87.2)$ & 0.829 \\
\hline \multicolumn{4}{|l|}{ Clinical symptoms } \\
\hline Fever & $9(69.2)$ & $30(63.8)$ & 0.974 \\
\hline Rash & $7(53.9)$ & $28(59.6)$ & 0.802 \\
\hline Photosensitivity & $4(30.8)$ & $9(19.2)$ & 0.603 \\
\hline Oral ulcer & $4(30.8)$ & $5(10.6)$ & 0.174 \\
\hline Arthritis & $4(30.8)$ & $7(14.9)$ & 0.366 \\
\hline Serositis & $5(38.5)$ & $20(42.6)$ & 0.791 \\
\hline Kidney involvement & $9(69.2)$ & $21(44.7)$ & 0.117 \\
\hline Hematologic & $12(92.3)$ & $36(76.6)$ & 0.389 \\
\hline \multicolumn{4}{|l|}{ involvement } \\
\hline Neurological complication & 0 & $10(21.3)$ & 0.161 \\
\hline
\end{tabular}


Number of cases with decreased

\begin{tabular}{|c|c|c|c|}
\hline \multirow{2}{*}{\multicolumn{4}{|c|}{$44(93.6)$}} \\
\hline & & & \\
\hline CMV pp65-positive & $12(92.3)$ & $33(70.2)$ & 0.205 \\
\hline CMV IgM-positive & $8(61.5)$ & $21(44.7)$ & 0.174 \\
\hline CMV DNA-positive & $1(7.7)$ & 0 & 0.217 \\
\hline SLEDAI-2000 score (0-4 points) & 0 & $2(4.3)$ & 1 \\
\hline SLEDAI-2000 score (5-9 points) & $2(15.4)$ & $9(19.2)$ & 0.925 \\
\hline \multicolumn{4}{|c|}{ SLEDAI-2000 score $\quad(10-14$} \\
\hline \multicolumn{4}{|l|}{ points) } \\
\hline SLEDAI-2000 $\quad$ score $\quad(\geq 15$ & $9(69.2)$ & $26(55.3)$ & 0.367 \\
\hline points) & & & \\
\hline
\end{tabular}

SLE treatment

Glucocorticoid

Pulse therapy

$3(23.1)$

$13(27.7)$

0.981

High-dose

glucocorticoid

$9(69.2)$

$18(38.3)$

0.086

therapy 


\begin{tabular}{|c|c|c|c|}
\hline Low-dose glucocorticoid therapy & $5(38.5)$ & $10(21.3)$ & 0.366 \\
\hline Unused glucocorticoid treatment & $1(7.7)$ & $1(2.1)$ & 0.389 \\
\hline Hydroxychloroquine sulfate & $10(76.9)$ & $39(82.9)$ & 0.925 \\
\hline Mycophenolate mofetil & $3(23.1)$ & $7(14.9)$ & 0.779 \\
\hline Cyclophosphamide & $2(15.4)$ & $5(10.6)$ & 0.987 \\
\hline Cyclosporine A & 0 & $4(8.5)$ & 0.568 \\
\hline Leflunomide & 0 & $1(2.1)$ & 1 \\
\hline Methotrexate & $1(7.7)$ & $2(4.3)$ & 0.526 \\
\hline Elamode & $1(7.7)$ & 0 & 0.217 \\
\hline Thalidomide & $1(7.7)$ & $2(4.3)$ & 0.526 \\
\hline Glucocorticoid combined & $5(38.5)$ & $16(34.0)$ & 0.974 \\
\hline immunosuppressive agent & & & \\
\hline
\end{tabular}

${ }^{*} P<0.05$ was considered significant. 